\title{
CARCINOMA DA GLÂNDULA SUPRA-RENAL
}

\author{
Adrenal gland carcinoma
}

\author{
Alexandre Coutinho Teixeira de FREITAS
}

ABCDDV/557

Freitas ACT. Carcinoma da glândula supra-renal. ABCD Arq Bras Cir Dig 2007; 20(3):195-200.

RESUMO - Racional - A neoplasia da glândula supra-renal pode ser oriunda de diversos tipos histológicos. O carcinoma é raro, correspondendo à $0,02 \%$ de todas as neoplasias. Objetivos - Revisar os últimos avanços relacionados ao diagnóstico e ao tratamento do carcinoma da glândula supra-renal. Métodos - Foi realizada revisão da literatura no Medline, livros texto de autores consagrados e referências obtidas de artigos considerados de maior relevância. Conclusães - Aproximadamente 79\% dos carcinomas supra-renais são funcionantes. O hormônio mais comumente secretado é o cortisol causando síndrome de Cushing. Portadores de lesões não-funcionantes podem apresentar queixas relacionadas com o crescimento local da lesão. Ele está presente em uma série de síndromes neoplásicas de origem familiar. Ainda não está definido se o carcinoma esporádico tem origem em lesões benignas. De acordo com os sintomas, a investigação diagnóstica inicial envolve dosagens de cortisol urinário e nível sérico de aldosterona e renina. A tomografia abdominal ou a ressonância magnética são os exames de imagem de primeira linha. O PET scan com fluorodeoxiglicose apresenta potencial para diferenciação entre lesão benigna e maligna. A biópsia com agulha fina não está indicada devido à sua alta taxa de complicações. O tratamento de eleição é o cirúrgico com ressecção em bloco de órgãos adjacentes se necessário. Linfadenectomia aórtica e retroperitoneal deve ser realizada. Recorrência local ou metástases ocorrem em $80 \%$ dos casos. Procedimentos cirúrgicos citoredutores beneficiam os casos de doença avançada. Na presença de metástases, nos submetidos à cirurgia citoredutora e nos com recorrência local à quimioterapia com mitotano está indicada. Radioterapia é o tratamento de escolha no caso de metástases ósseas e tratamento adjuvante em alguns casos de risco elevado para recorrência. Em adultos a sobrevida média geral em 5 anos varia de $10 \%$ a $35 \%$. Após operação curativa varia de $20 \%$ a $58 \%$.

DESCRITORES - Neoplasias das glândulas supra-renais. Diagnóstico. Cirurgia.

\section{INTRODUÇÃO}

As neoplasias das glândulas supra-renais são oriundas de diversos tipos histológicos. Apresentam manifestação clínica variada, dependendo da produção ou não de hormônios. As neoplasias do córtex podem secretar corticosteróides, aldosterona ou esteróides sexuais. $\mathrm{O}$ hormônio secretado pode variar em um mesmo tipo histológico. Por exemplo, na maioria das situações os carcinomas secretam corticosteróides, porém, podem secretar aldosterona.

$\mathrm{O}$ carcinoma supra-renal é raro, correspondendo à $0,02 \%$ de todas as neoplasias. Sua incidência é de um a dois casos por 1,7 milhão da população e é discretamente mais freqüente em mulheres ${ }^{23}$. A incidência é maior em crianças com menos de 5 anos e na $4^{\text {a }}$ e $5^{\text {a }}$ décadas de vida ${ }^{23}$.

Esse estudo tem por objetivo revisar os últimos avanços relacionados ao diagnóstico e ao tratamento do carcinoma da glândula supra-renal.

Trabalho realizado no Serviço de Cirurgia do Aparelho Digestivo do Hospital de Clínicas da Universidade Federal do Paraná, Curitiba, PR, Brasil.

Endereço para correspondência: Alexandre Coutinho Teixeira de Freitas, e-mail: alexandrefreitas@ufpr.br

\section{MÉTODO}

As palavras-chave "adrenal carcinoma" e "adrenal gland neoplasms" foram pesquisadas no Medline em artigos publicados nos últimos 10 anos. Em relação ao tipo de estudo foram selecionados artigos de revisão, metanálises, estudos randomizados controlados, estudos clínicos e protocolos. Somente foram obtidos artigos em seres humanos na língua inglesa. Essa pesquisa forneceu: 320 artigos (242 de revisão) para o termo "adrenal carcinoma" e 742 artigos (598 de revisão) para o termo "adrenal gland neoplasms". Os trabalhos considerados de maior relevância foram selecionados e analisados. Também foram analisados livros-texto de autores consagrados na área e outras publicações selecionadas das referências bibliográficas dos artigos obtidos na pesquisa no Medline e considerados de maior interesse.

\section{REVISÃO DA LITERATURA}

Os carcinomas da glândula supra-renal são funcionantes em $79 \%$ dos $\operatorname{casos}^{5,33}$. O tipo de hormônio produzido pode modificar de acordo com o tamanho da lesão, seu grau de diferenciação e crescimento. O hormônio mais comumente secretado é o cortisol causando síndrome de Cushing ${ }^{7,12}$. Os sintomas de atrofia de pele, fraqueza 
muscular, hiperglicemia, hipertensão arterial e alterações psiquiátricas comumente apresentam aparecimento súbito e rápida evolução ${ }^{26}$. Produção de androgênios em mulheres desencadeia hirsutismo, atrofia mamária, voz grave e alterações menstruais ${ }^{26}$. Em homens, carcinomas secretores de estrogênios causam ginecomastia e atrofia testicular; em mulheres, causam sensibilidade mamária e alterações menstruais ${ }^{26}$. Produção de aldosterona (aldosteronismo primário) é rara ${ }^{7,12,21}$. É controverso se tumores funcionantes apresentam melhor ou pior prognóstico ${ }^{4}$.

Doentes com lesões não funcionantes podem apresentar queixas relacionadas com o crescimento local da lesão, como: dor abdominal, distensão, náuseas e vômitos ${ }^{5}$. De acordo com o tamanho das lesões, o exame clínico pode evidenciar massa palpável. A apresentação inicial pode estar relacionada à doença metastática, como fraturas patológicas. Observa-se incremento significativo do número de casos detectados incidentalmente (incidentalomas) em exames de imagem indicados por outras causas ${ }^{13}$. Nesse caso, a conduta a ser tomada depende de vários fatores $\mathrm{e}$ será abordada no final desse artigo.

A síndrome de Cushing é a manifestação clínica dos tumores com produção primária ou secundária de corticosteróides. Em $75 \%$ a $80 \%$ dos casos têm origem central (doença de Cushing), no hipotálamo ou na hipófise, em tumores produtores de CRH e ACTH, respectivamente; em $10 \%$ a $15 \%$ dos casos têm origem ectópica, como nas neoplasias brônquicas e tumores carcinóides produtores de CRH e ACTH; em $20 \%$ a $30 \%$ dos casos têm origem no córtex da glândula supra-renal, em tumores produtores de corticosteróides ${ }^{22}$. Entre os tumores primários produtores de corticosteróides, $10 \%$ a $15 \%$ são adenomas; $5 \%$ a $10 \%$ são carcinomas e aproximadamente $5 \%$ são devidos a hiperplasia ${ }^{22}$.

O aldosteronismo primário é definido como estado de excesso de produção de aldosterona e subseqüente supressão dos níveis séricos de renina ${ }^{20}$. Em $2 / 3$ dos casos a etiologia é a hiperplasia bilateral da supra-renal, em $1 / 3$ dos casos adenomas ${ }^{20,25}$. O carcinoma, o aldosteronismo familiar tipo I e tipo II e a utilização de carbenoxolona são causas $\operatorname{raras}^{25}$. O aldosteronismo primário habitualmente acomete pessoas na faixa entre 40 e 50 anos de idade com prevalência discretamente maior em mulheres ${ }^{20}$. Com freqüência, os doentes são assintomáticos ou apresentam queixas relacionadas à hipertensão arterial como cefaléia. A hipertensão arterial apresenta a característica de ser refratária ao tratamento clínico. Estima-se que 5\% a $12 \%$ de todos os doentes com hipertensão arterial apresentem aldosteronismo primário ${ }^{20,25}$. Os níveis de potássio sérico estão diminuídos em $1 / 3$ dos $\operatorname{casos}^{20,25}$.

\section{Gênese tumoral}

O carcinoma supra-renal está presente em uma série de síndromes neoplásicas de origem familiar. Nesses casos, foram demonstradas diversas alterações moleculares genéticas: na síndrome de Li-Fraumeni, alterações no gene p53 no locus 17p13; na neoplasia endócrina múltipla do tipo
1, alterações no gene menin no locus 11q13; na síndrome de Carney, perda da heterogeneidade no locus 2p16; e na síndrome de Beckwith-Wiedemann, alterações de IGF2 (insulin-like growth factor 2) no locus $11 \mathrm{p} 15.5^{5,23}$. As principais características dessas síndromes estão representadas no Quadro 1.

QUADRO 1 - Síndromes neoplásicas familiares com carcinoma da supra-renal

\begin{tabular}{|c|c|c|}
\hline Síndrome & Característica & Lesões associadas \\
\hline Li-Fraumeni & $\begin{array}{l}\text { Autossômica } \\
\text { dominante }\end{array}$ & $\begin{array}{l}\text { Tumores de mama, sarcoma de partes } \\
\text { moles, tumores cerebrais, osteosar- } \\
\text { coma e leucemia }\end{array}$ \\
\hline $\begin{array}{l}\text { Neoplasia endócrina } \\
\text { múltipla do tipo } 1\end{array}$ & $\begin{array}{l}\text { Autossômica } \\
\text { dominante }\end{array}$ & $\begin{array}{l}\text { Tumores de paratireóide, pâncreas } \\
\text { endócrino, duodeno e hipófise an- } \\
\text { terior }\end{array}$ \\
\hline Carney & $\begin{array}{l}\text { Autossômica } \\
\text { dominante }\end{array}$ & Mixomas, pigmentação cutânea \\
\hline Beckwith-Wiedemann & $\begin{array}{l}\text { Autossômica } \\
\text { dominante }\end{array}$ & $\begin{array}{l}\text { Tumor de Wilms, hepatoblastoma, } \\
\text { rabdomiosarcoma }\end{array}$ \\
\hline
\end{tabular}

Ainda não está definido se o carcinoma supra-renal esporádico tem origem em lesões benignas como os adenomas ou a hiperplasia ou trata-se de doença distinta. Existem relatos de lesões benignas com evolução para carcinoma. Estudos mostram associação de carcinoma supra-renal com elevação de IGF1 (insulin-like growth factor 1) e IGF2, fatores normalmente associados à diferenciação do córtex da glândula ${ }^{4}$. Sua elevação pode estar associada à gênese e diferenciação tumoral. Estudo em murinos demonstrou que o gene SCCRO (squamous cell carcinoma-related oncogene), apresentou expressão elevada nos casos de lesões benignas como o adenoma ${ }^{3}$. Em $65 \%$ dos casos de carcinoma existiu perda de sua expressão. Outro estudo, em seres humanos, demonstrou elevação de VEGF (vascular endotelial growth factor), um marcador de angiogênese, nos casos de adenoma e hiperplasia ${ }^{2}$. Nos casos de carcinoma, a elevação foi superior à observada nos casos de lesões benignas.

\section{Diagnóstico}

A investigação inicial depende da apresentação clínica. Pacientes com sintomas de síndrome de Cushing devem ser inicialmente investigados com dosagem de cortisol na urina de 24 horas (valor normal de 90 a $100 \mathrm{ug} / 24$ horas) ${ }^{22}$. A diferenciação entre elevação do cortisol dependente ou independente do ACTH pode ser realizado pela dosagem de cortisol sérico e ACTH sérico no período noturno ${ }^{23}$. Se o nível de cortisol for acima de $50 \mathrm{ug} / \mathrm{dL}$ e o de ACTH inferior a $5 \mathrm{pg} / \mathrm{mL}$, a produção de cortisol é independente do ACTH, indicando origem na glândula supra-renal. Se o nível de ACTH for superior a $50 \mathrm{pg} / \mathrm{mL}$, a produção de cortisol é dependente do ACTH, indicando origem na hipófise ou produção ectópica. Casos duvidosos podem ser investigados com o teste de supressão com a dexametasona ( $2 \mathrm{mg}$ a cada 6 horas por 2 dias $)^{22}$. Nesse teste não há diminuição do cortisol sérico ou urinário nos casos de carcinoma, adenoma ou produção ectópica de ACTH sendo 
observados valores superiores a 5 ug/dL. Redução de 50\% em relação ao nível basal indica origem na hipófise.

Investigação para aldosteronismo está indicada nos doentes com hipertensão arterial e hipocalemia; nos com hipertensão arterial com necessidade de três ou mais medicamentos anti-hipertensivos e com níveis pressóricos superiores a 140/90 mmHg; nos casos de história familiar de hipertensão arterial; na deteç̧ão incidental de massa adrenal e nos casos de hipocalemia induzida por tiazídi$\cos ^{25}$. Alguns autores recomendam a investigação para qualquer paciente hipertenso. $\mathrm{O}$ teste inicial é a dosagem do nível sérico aldosterona/renina ${ }^{25}$. O valor de corte para o diagnóstico varia de 20 a $50 \mathrm{ng} / \mathrm{dL}: \mathrm{ng} / \mathrm{ml} / \mathrm{h}$, dependendo do método de dosagem utilizado. Como muitos pacientes apresentam normocalemia, a dosagem do potássio sérico é ineficaz. Teste confirmatório é necessário nos casos em que o teste inicial foi positivo. O padrão-ouro é o teste da supressão com fluodrocortisona, administrada na dose de $0,1 \mathrm{mg}$ a cada 6 horas em associação com cloreto de sódio de liberação lenta na dose de $1,75 \mathrm{~g}$ a cada 12 horas $^{25}$. Esses teste apresenta risco de hipocalemia severa e crise hipertensiva necessitando de 5 dias de internação. Nível de aldosterona superior a $6 \mathrm{ng} / \mathrm{mL}$, indicando supressão incompleta, confirma o diagnóstico de hiperaldosteronismo primário. Podem ser realizados testes mais simples, em nível ambulatorial, como a supressão da aldosterona com infusão de solução salina isotônica via endovenosa ou a supressão com a administração de sódio via oral ${ }^{25}$.

A tomografia abdominal é o exame de imagem de primeira linha para investigação ${ }^{23}$. Os carcinomas de supra-renal podem mimetizar os adenomas, porém geralmente aqueles apresentam margens irregulares e diâmetro maior que $5 \mathrm{~cm}$. O tamanho da lesão é o principal fator indicativo de malignidade. Estudo com 223 casos mostrou, no momento do diagnóstico, diâmetro médio de $12 \pm 6 \mathrm{~cm}^{12}$. A probabilidade do diagnóstico de carcinoma supra-renal aumenta de $35 \%$ a $98 \%$ em portadores de lesões com diâmetro superior a 6 $\mathrm{cm}^{1}$. Em algumas situações, tanto os carcinomas quanto os feocromocitomas apresentam necrose e degeneração cística, devendo ser pesquisados em termos de produção hormonal e malignidade ${ }^{23}$. Cerca de $2 \%$ dos carcinomas supra-renais são pseudocistos ${ }^{6}$. A diferenciação entre adenomas, carcinomas, feocromocitomas e metástases para supra-renal pode ser realizada através da tomografia sem contraste seguida de exame com contraste e análise retardada após 1 e 10 minutos $^{28}$. Na tomografia inicial, sem contraste, os adenomas apresentam taxa de atenuação média inferior aos carcinomas, feocromocitomas e metástases. Um minuto após a administração do meio de contraste, observa-se redução de $50 \%$ na taxa de atenuação e após 10 minutos redução de $40 \%$. Nessa situação, a sensibilidade e especificidade para os adenomas de supra-renal são de $100 \%$.

A ressonância magnética e o PET scan também podem ser usados para diferenciar lesões benignas e malignas. $\mathrm{Na}$ ressonância magnética os carcinomas tendem a ser hipointensos em $\mathrm{T} 1$ e hiperintensos em $\mathrm{T} 2$ em relação ao fígado ou ao baço ${ }^{29}$. Sua eficácia é semelhante à da tomografia $^{1}$. O uso de gadolíneo melhora significativamente a caracterização das lesões adrenais apresentando captação distinta e lavagem lenta ${ }^{1}$.

O PET scan tem apresentado bons resultados para diferenciação entre lesão benigna e maligna. Diversos agentes têm sido usados, entre eles a fluorodeoxiglicose (FDG) e o metomidate (MTO). A fluorodeoxiglicose é captada e detectada somente nos casos de lesões malignas ${ }^{33}$. O metomidate não diferencia lesão benigna de lesão maligna, porém demonstra se o tumor é do córtex ou medula da glândula supra-renal ${ }^{14}$. A maioria dos estudos publicados é com pequeno número de casos. Estudos com maior número de casos são necessários para estabelecer a real eficácia do PET scan nos tumores adrenais. Além disso, sua disponibilidade é limitada e o custo elevado.

A ultra-sonografia pode ser usada para seguimento e acompanhamento do tamanho das lesões ${ }^{23}$. É o método de screening de eleição para gestantes e pacientes pediátri$\cos ^{23}$. Aparelhos modernos com reconstrução das imagens em 3 ou 4 dimensões apresentam acurácia superior à da tomografia e ressonância magnética para demonstrar invasão de estruturas adjacentes.

A biópsia com agulha fina não está indicada devido à sua alta taxa de complicações. Elas ocorrem em aproximadamente $12 \%$ dos casos, especialmente implante de metástases no trajeto da agulha ${ }^{1}$. Deve-se levar em conta ainda o seu controverso valor diagnóstico ${ }^{1}$.

Para o estadiamento do carcinoma supra-renal recomenda-se a realização de tomografia abdominal e de tórax de alta resolução. O PET scan com fluorodeoxiglicose pode ser usado para diferenciar possíveis metástases de lesões benignas. Cintilografia óssea deve ser realizada no momento do diagnóstico ou no caso de queixas de dores ósseas. As áreas com captação aumentada devem ser investigadas com exame radiológico convencional. A dosagem hormonal é importante para o estadiamento no período pós-operatório. Após o tratamento esperam-se níveis de cortisol subnormais. Se após um procedimento potencialmente curativo, forem observados níveis elevados de cortisol, deve-se, considerar o paciente em estádio IV (vide estadiamento a seguir), mesmo na ausência de metástases detectáveis em exames de imagem.

\section{Tratamento}

O tratamento de eleição é a ressecção cirúrgica do tumor e da glândula supra-rena ${ }^{15}$. A operação deve ser individualizada de acordo com o estadiamento. Diversos modelos foram propostos. O estadiamento de Lee et al. ${ }^{17}$ está representado no Quadro 2. No momento do diagnóstico 87\% dos casos em adultos apresentam doença avançada (estádios 3 e 4). Metástases ocorrem com mais freqüência para fígado (44\% a 93\%), pulmão (46\% a 79\%), linfonodos à distância (18\%-73\%), e peritônio (16\%-79\%) $)^{11,16,18}$.

Na presença de invasão de órgãos adjacentes é recomendada a ressecção em bloco com o rim, o pâncreas, parte do fígado ou o baço ${ }^{12}$. Linfadenectomia aórtica e retroperitonial deve ser realizada. A presença de trombo na veia renal ou na veia cava inferior não impede a realização de cirurgia 
QUADRO 2 - Estadiamento do carcinoma supra-renal ${ }^{17}$

\begin{tabular}{|cl|}
\hline Estádio & \\
\hline 1 & $<5 \mathrm{~cm}$, sem invasão local, metástases a distância ou para linfonodos \\
2 & $>5 \mathrm{~cm}$, sem invasão local, metástases a distância ou para linfonodos \\
3 & Presença de invasão local ou metástases para linfonodos \\
4 & Presença de metástases a distância \\
\hline
\end{tabular}

curativa, no entanto tecnicamente pode ser necessário o uso de bypass cardíaco ${ }^{10}$. Nos casos de doença avançada, sem potencial curativo, os pacientes podem se beneficiar de operação citoredutora que promove diminuição da produção hormonal e melhora da qualidade de vida.

A adrenalectomia apresenta índices de morbidade entre dois e $10 \%$ e a mortalidade varia de um a $5 \%{ }^{12}$. O procedimento pode ser realizado por diversos acessos ${ }^{22}$. $\mathrm{O}$ acesso anterior, transabdominal, é o mais utilizado ${ }^{22}$. É realizado através de incisão abdominal subcostal unilateral ou bilateral ou mediana. Esse acesso permite palpação da glândula contra-lateral e inspeção da cavidade abdominal para verificação de metástases. O acesso tóraco-abdominal pode ser utilizado no caso de lesões grandes. $\mathrm{O}$ acesso posterior com ressecção da $12^{\text {a }}$ costela somente é utilizado para lesões benignas pequenas.

Atualmente a cirurgia laparoscópica é a via de escolha para realização de adrenalectomia em lesões benignas funcionantes ou não-funcionantes até $12 \mathrm{~cm}$ de diâmetro ${ }^{22}$. A cirurgia minimamente invasiva apresenta vantagens em relação à aberta em termos de menor necessidade de analgésicos, menor tempo de internação, maior satisfação do paciente e retorno mais rápido às atividades normais. O acesso laparoscópico não está indicado no caso de carcinoma supra-renal devido à possibilidade de violação da cápsula tumoral, fragmentação tumoral e dificuldade técnica de se obter ressecção total da lesão com margens cirúrgicas livre e dissecção adequada de linfonodos ${ }^{1,5}$.

Mesmo após a remoção cirúrgica, a diferenciação entre lesões benignas e malignas pode ser difícil. O peso da lesão é parâmetro importante. Os adenomas pesam, em média, entre 20 e $50 \mathrm{~g}$, os carcinomas habitualmente apresentam peso maior, ao redor de $100 \mathrm{~g}^{24}$. Diversos escores diagnósticos foram propostos, considerando os marcadores histopatológicos típicos, como: número elevado de mitoses, mitoses atípicas, invasão vascular, invasão da cápsula da glândula e necrose ${ }^{1}$. Marcadores moleculares têm sido estudados, porém nenhum marcador isoladamente é diagnóstico de carcinoma supra-renal ${ }^{1}$.

Após operação curativa, cerca de $80 \%$ dos pacientes evoluem com recorrência local ou metástases à distância. Fatores de risco são: lesão estádio III, lesão com diâmetro superior a $12 \mathrm{~cm}$, hemorragia intra-mural e índice elevado de mitoses. No caso de recorrência local a reoperação traz benefícios em termos de aumento de sobrevida e cura pode ser obtida em casos esporádicos ${ }^{27}$. A sobrevida em 5 anos dos pacientes reoperados é de $50 \%$; os submetidos à quimioterapia isolada apresentam sobrevida de $8 \%{ }^{27}$. Em casos selecionados, o tratamento cirúrgico deve ser considerado no caso de metástase única para fígado, pulmão e cérebro ${ }^{27}$.
Quimioterapia adjuvante apresenta efeitos limitados. O quimioterápico mais utilizado é o mitotano com resposta terapêutica em $14 \%$ a $35 \%$ dos $\operatorname{casos}^{15,30}$. A maioria dos estudos não mostra benefícios de sua utilização no caso de operação curativa ${ }^{12}$. Nos casos de doença avançada metastática (estádio 4), nos pacientes submetidos à operação citoredutora e nos com recorrência local, a quimioterapia com mitotano está indicada em associação com a operação. Estudos mostram aumento da sobrevida média de 2 para 4 anos quando comparados com pacientes submetidos à operação isoladamente ${ }^{12}$. Outros quimioterápicos podem ser utilizados em conjunto com o mitotano. Estudos multicêntricos são necessários para comprovação de sua efetividade.

Radioterapia está indicada em algumas situações. É o tratamento de escolha no caso de metástases ósseas e tratamento adjuvante em alguns casos de risco elevado para recorrência. Estudos com número pequeno de casos sugerem aumento da sobrevida e diminuição da taxa de recorrência local com irradiação de 50 a $60 \mathrm{~Gy}$ no leito tumoral no caso de lesões com diâmetro superior a $12 \mathrm{~cm}$, índice mitótico elevado, violação da cápsula tumoral e fragmentação tumoral ${ }^{19}$.

O seguimento é fundamental após o tratamento, principalmente o curativo, devido às altas taxas de recorrência. Normalmente é negligenciado nos pacientes operados com lesões nos estádios 1 e 2 devido à presunção de cura. Estudos mostram recorrência local mesmo nessas situações ${ }^{1,12}$. Nos tumores funcionantes o seguimento deve ser realizado com dosagem de cortisol na urina a intervalos regulares. No caso de recorrência, a elevação dos níveis hormonais precede a detecção de lesões em exames de imagem. Estes, como a tomografia ou ressonância magnética, devem ser realizados inicialmente a cada 3 a 4 meses nos 2 primeiros anos após a ressecção ${ }^{1}$. A partir desse período, os intervalos podem ser progressivamente aumentados.

Em pacientes pediátricos com idade inferior a 5 anos a sobrevida média geral em 5 anos é de $49 \%{ }^{23}$. Nos casos de operação curativa a sobrevida em 5 anos é de $70 \%$. Em adultos a sobrevida média geral em 5 anos varia de $10 \%$ a $35 \%{ }^{23}$. Após operação curativa, varia de $20 \%$ a $58 \%$. No estádio 1 a sobrevida em 5 anos é de $50 \%$; nos estádios 2 e 3 ela é de $30 \%$ e no estádio 4 , de $10 \%$ a $20 \%$.

\section{Incidentaloma}

$\mathrm{O}$ advento de exames de imagem como a tomografia computadorizada, permite o achado incidental de lesões nodulares nas glândulas supra-renais em $0,4 \%$ a 4,4\% dos $\operatorname{casos}^{32}$. Nessa situação, duas questões devem ser abordadas e serão importantes para definição da conduta: a) se o nódulo apresenta atividade hormonal; b) se ele é lesão maligna ou benigna. Na maioria dos casos os incidentalomas são adenomas benignos, não funcionantes ${ }^{31}$. Outros possíveis diagnósticos incluem: adenomas secretores de cortisol em 5,3\% dos casos, adenomas secretores de aldosterona (aldosteronomas) em 1\%, carcinomas em 
$4,7 \%$, feocromocitomas em $5 \%$ e metástases de outras neoplasias em 2,5\% ${ }^{31}$. Tumores secretores de hormônios sexuais são raros.

Inicia-se a avaliação com história clínica e exame físico detalhados com foco em sinais e sintomas relacionados à secreção hormonal e doença maligna ${ }^{32}$. Deve-se realizar busca ativa em relação à hipertensão arterial, perda de peso, astenia, síndrome de Cushing, virilização, feminização, alterações do ciclo menstrual e evidências de doença maligna oculta (pesquisa de sangue oculto nas fezes, anemia, exame de Papanicolau). A verificação de atividade hormonal deve ser realizada através dos níveis séricos de potássio e renina (diagnóstico de aldosteronoma) e da coleta de urina de 24 horas para determinação de cortisol (diagnóstico de síndrome de Cushing), ácido vanil-mandélico e catecolaminas (diagnóstico de feocromocitoma). Alguns pacientes podem apresentar síndrome de Cushing subclínica, ou seja, não apresentam os estigmas clínicos clássicos, porém apresentam elevação da secreção de cortisol $^{32}$. Nessa situação, a dosagem de cortisol na urina de 24 horas pode ser normal. Logo, indica-se também a realização de teste de supressão com dexametasona em todos os casos. Cortisol sérico acima de $5 \mathrm{ug} / \mathrm{dL}$ indica produção autônoma. Sua especificidade é de $91 \%{ }^{8}$. Não há indicação de pesquisa hormonal relacionada a tumores produtores de hormônios sexuais ${ }^{32}$.

O tamanho da lesão é outro fator importante na avaliação. Habitualmente, lesões malignas apresentam diâmetro superior a $6 \mathrm{~cm}$. No entanto, lesões menores também podem ser malignas. Existe recomendação na literatura para ressecção de qualquer lesão maior que $4 \mathrm{~cm}^{9,32}$. Nessa decisão devem ser levados em conta o aspecto da lesão nos exames de imagem e dados clínicos do paciente como a idade. As lesões menores que $4 \mathrm{~cm}$ devem ser ressecadas nas situações de produção hormonal. Como ocorre na maioria das neoplasias endócrinas, a biópsia com agulha não permite diferenciação entre lesão benigna e maligna ${ }^{1}$. Nas lesões não-funcionantes menores que $4 \mathrm{~cm}$ com história pregressa de outra neoplasia, a biópsia está indicada para diagnóstico de implante metastático secundário na glândula supra-renal ${ }^{9,32}$. Nas situações sem história pregressa de neoplasia, a biópsia não é realizada e deve-se repetir a tomografia em 6, 12 e 24 meses $^{32}$. Procedimento cirúrgico está indicado se houver crescimento da lesão. Existem evidências que lesões não-funcionantes podem tornar-se funcionantes após 4 anos de seguimento, logo indica-se a pesquisa hormonal anualmente durante esse período 9 . A conduta diagnóstica e terapêutica do incidentaloma está representada no algoritmo da Figura 1.

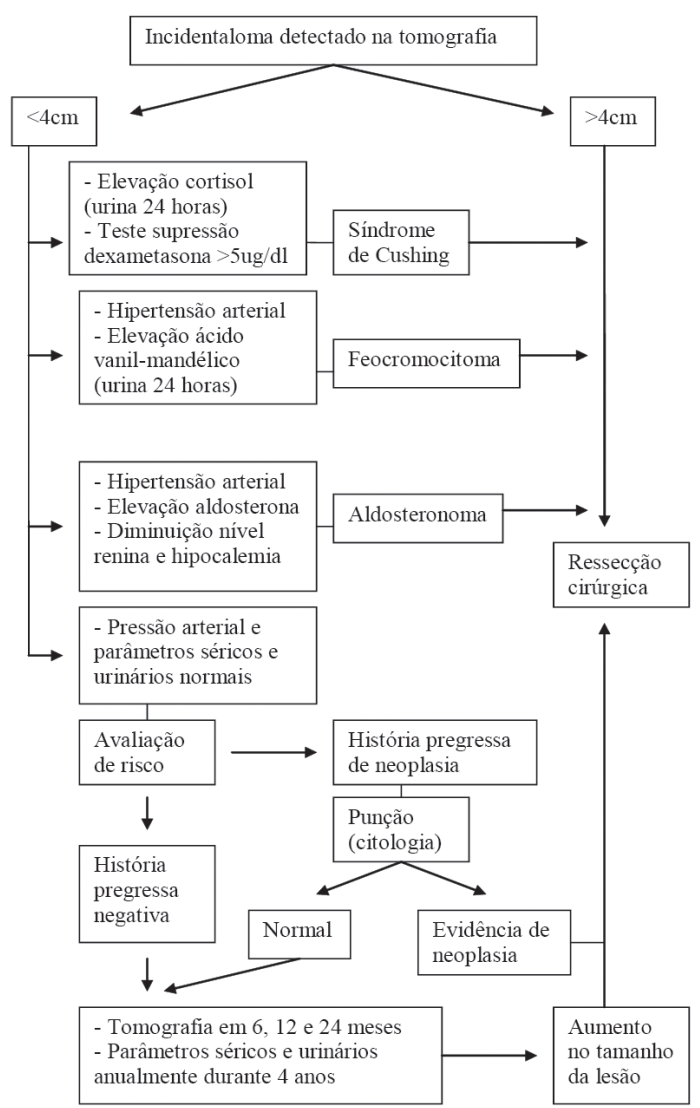

FIGURA 1 - Conduta diagnóstica e terapêutica do incidentaloma

\section{CONCLUSÕES}

O diagnóstico de carcinoma supra-renal é baseado em suas características na tomografia e na ressonância magnética. O PET scan apresenta bons resultados em termos de diferenciação entre lesões benignas e malignas. Estudos prospectivos com número maior de casos são necessários para estabelecer sua acurácia. A biópsia com agulha fina não deve ser realizada devido ao risco de complicações e dificuldade de diagnóstico. O tratamento curativo é através da ressecção cirúrgica com dissecção de linfonodos aórticos e retroperitoniais. A ressecção de órgãos adjacentes pode ser necessária. Recorrência local ocorre em até $80 \%$ dos casos. Operação citoredutora beneficia pacientes com doença avançada. Quimioterapia com mitotano está indicada nos casos de doença avançada metastática, nos pacientes submetidos à operação citoredutora e nos pacientes com recorrência local. O seguimento deve ser realizado com dosagem hormonal e exames de imagem. 
Freitas ACT. Adrenal gland carcinoma. ABCD Arq Bras Cir Dig 2007; 20(3):195-200.

ABSTRACT - Background - Adrenal gland neoplasm may be originated from diverse histological types. Carcinomas are rare findings, and correspond to only $0,02 \%$ of all neoplasms. Aim - To review the latest advances in relation to the treatment of adrenal gland carcinomas. Methods - A literature review was performed using Medline, text books and authors, as well as references obtained from relevant articles. Conclusion - Approximately $79 \%$ of adrenal gland carcinomas are functional. The most commonly secreted hormone is cortisol, which causes Cushing's syndrome. Patients with non-functional lesions may complain about local growth of the lesion. This type of carcinoma is present in a series of other neoplasic syndromes of familiar origen. According to the symptoms, initial diagnostic investigation involves dosage of urinary cortisol, as well as aldosterone and renin serum levels. Abdominal tomography or magnetic ressonance are first class image tests. Fluorodeoxyglucose pet scan is a tool that can be used to differentiate benign and malignant lesions. Fine needle biopsy is not indicated due to the high rate of complications. The choosen treatment is usually surgery with block ressection of adjacent organs if necessary. Aortic and retroperitoneal lymphadenectomy should be performed. Local recurrence and metastasis occur in $80 \%$ of the cases. Cytoreductive surgical procedures benefit cases of advanced disease. Quimiotherapy using mitotane is indicated to patients who were submitted to cytoreductive surgery, who have had local recurrence and in those with metastasis. Radiotherapy is the treatment of choice in the event of bone metastasis and adjuvant treatment is used in a few cases with elevated recurrence risks. In adults, the overall average life span in 5 years varies between $10 \%$ to $35 \%$. After curative surgery it varies between $20 \%$ to $58 \%$.

HEADINGS - Adrenal glands neoplasms. Diagnosis. Surgery

\section{REFERÊNCIAS}

1. Allolio B, Hahner S, Weismann D, Fassnacht M. Management of adrenocortical carcinoma. Clin Endocrinol. 2004;60:273-87.

2. Beuschlein F, Looyenga BD, Reincke M, Hammer GD. Role of inhibin/activin system and luteinizing hormone in adrenocortical tumorigenesis. Horm Metab Res. 2004;36:392-6.

3. Boulle N, Logie A, Gicquel C, Perin L, Le Bouc Y. Increased levels of insulin-like growth factor II (IGF-II) and IGF-binding protein-2 are associated with malignancy in sporadic adrenocortical tumors. J Clin Endocrinol Metab. 1998;83:1713-8.

4. Chen DY, Sosa RE, Scherr DS. Treatment of adrenocortical carcinoma: contemporary outcomes. Curr Urol Rep. 2004;5:65-72.

5. Dackiw AP, Lee JE, Gagel RF, Evans DB. Adrenal cortical carcinoma. World J Surg. 2001;25:914-26.

6. Erickson LA, Lloyd RV, Hartman R, Thompson G. Cystic adrenal neoplasms. Cancer. 2004;101:1537-44.

7. Favia G, Lumachi F, D'Amico DF. Adrenocortical carcinoma: is prognosis different in nonfunctioning tumors? Results of surgical treatment in 31 patients. World J Surg. 2001;25:735-8.

8. Gorges R, Knappe G, Gerl H, Ventz M, Stahl F. Diagnosis of Cushing's syndrome: re-evaluation of midnight plasma cortisol vs urinary free cortisol and low-dose dexamethasone suppression test in a large patient group. J Endocrinol Invest. 1999;22:241-9.

9. Grumbach MM, Biller BM, Braunstein GD, Campbell KK, Carney JA, Godley PA, Harris EL, Lee JK, Oertel YC, Posner MC, Schlechte JA, Wieand JA. Management of the clinically inapparent adrenal mass ("incidentaloma"). Ann Intern Med. 2003;138:424-9.

10. Hedican SP, Marshall FF. Adrenocortical carcinoma with intracaval extension. J Urol. 1997;158:2056-61.

11. Hutter AM Jr, Kayhoe DE. Adrenal cortical carcinoma. Results of treatment with op DDD in 138 patients. Am J Med 1966;41:581-92.

12. Icard P, Goudet P, Charpenay C, Andreassian B, Carnaille B, Chapuis Y, Cougard P, Henry JF, Proye C. Adrenocortical carcinomas: surgical trends and results of a 253-patient series from the French Association of Endocrine Surgeons study group. World J Surg. 2001;25:891-7.

13. Kasperlik-Zaluska AA, Migdalska BM, Makowska AM. Incidentally found adrenocortical carcinoma. A study of 21 patients. Eur J Cancer. 1998;34:17214.

14. Khan TS, Sundin A, Juhlin C, Langstrom B, Bergstrom M, Eriksson B. 11Cmetomidate PET imaging of adrenocortical cancer. Eur J Nucl Med Mol Imaging. 2003;30:403-10.

15. Khorram-Manesh A, Ahlman H, Jansson S, Wangberg B, Nilsson O, Jakobsson CE, Eliasson B, Lindstedt S, Tisell LE. Adrenocortical carcinoma: surgery and mitotane for treatment and steroid profiles for follow-up. World J Surg. 1998;22:605-11.

16. King DR, Lack EE. Adrenal cortical carcinoma: a clinical and pathologic study of 49 cases. Cancer. 1979;44:239-44.
17. Lee JE, Berger DH, el-Naggar A, Hickey RC, Vassilopoulou-Sellin R, Gagel RF, Burgess MA, Evans DB. Surgical management, DNA content, and patient survival in adrenal cortical carcinoma. Surgery. 1995;118:1090-8.

18. Luton JP, Cerdas S, Billaud L, Thomas G, Guilhaume B, Bertagna X, Laudat MH, Louvel A, Chapuis Y, Blondeau P, Bonnin A, Bricaire H. Clinical features of adrenocortical carcinoma, prognostic factors, and the effect of mitotane therapy. N Engl J Med. 1990;322:1195-201.

19. Markoe AM, Serber W, Micaily B, Brady LW. Radiation therapy for adjunctive treatment of adrenal cortical carcinoma. Am J Clin Oncol. 1991;14:170-4.

20. Meyer A, Brabant G, Behrend M. Long-term Follow-up after adrenalectomy for primary aldosteronism. World J Surg. 2005;29:155-9.

21. Mussig K, Wehrmann M, Horger M, Masser-Gluth C, Haring HU, Kaiserling E. Adrenocortical carcinoma producing 11-deoxycorticosterone: a rare cause of mineralocorticoid hypertension. J Endocrinol Invest. 2005;28:61-5.

22. Norton JA, Li M, Gillary J, Le HN. Cushing's Syndrome. Curr Probl Surg. 2001;38:487-545.

23. Roman S. Adrenocortical carcinoma. Curr Opin Oncol. 2006;18:36-42.

24. Saeger W. Histopathological classification of adrenal tumours. Eur J Clin Invest. 2000;30:58-62.

25. Schirpenbach C, Reincke M. Screening for primary aldosteronism. Best Pract Res Clin Endocrinol Metab. 2006;20:369-84.

26. Schteingart DE, Homan D. Management of adrenal cancer in adrenal disorders. New Jersey: Humana Press; 2001. p.231-48.

27. Schulick RD, Brennan MF. Long-term survival after complete resection and repeat resection in patients with adrenocortical carcinoma. Ann Surg Oncol. 1999;6:719-26.

28. Szolar DH, Korobkin M, Reittner P, Berghold A, Bauemhofer T, Trummer H, Schoellnast H, Preidler KW, Samonigg H. Adrenocortical carcinomas and adrenal pheochromocytomas: mass and enhancement loss evaluation at delay contrast-enhanced CT. Radiology. 2005;234:479-85.

29. Vaughan ED. Diseases of the adrenal gland. Med Clin N Am. 2004;88:44366.

30. Wooten MD, King DK. Adrenal cortical carcinoma. Epidemiology and treatment with mitotane and a review of the literature. Cancer. 1993;72:314555.

31. Young WF. Management approaches to adrenal incidentalomas: a view from Rochester, Minnesota. Endocrinol Metab Clin North Am. 2000;29:159-85.

32. Young WF. The incidentally discovered adrenal mass. N Engl J Med. 2007:356:601-10.

33. Zettinig G, Mitterhauser M, Wadsak W, Becherer A, Pirich C, Vierhapper H, Niederle B, Dudczak H, Kletter K. Positron emission tomography imaging of adrenal masses: FDG and the 11b-hydroxylase tracer $11 \mathrm{C}$ - metomidate. Eur J Nucl Med Mol Imaging. 2004;31:1224-30.

Conflito de interesse: não há Fonte financiadora: não há Recebido para publicação em: 04/04/2007 Aceito para publicação em: 15/05/2007 